\title{
The Relationship Between Peripheral Neuropathy Induced by Docetaxel and Systemic Inflammation-based Parameters in Patients with Breast Cancer
}

\author{
KOSHO YAMANOUCHI ${ }^{1}$, SAYAKA KUBA ${ }^{1}$, CHIKA SAKIMURA ${ }^{1}$, MICHI MORITA $^{1}$, KENGO KANETAKA $^{1}$, \\ KAZUMA KOBAYASHI $^{1}$, MITSUHISA TAKATSUKI ${ }^{1}$, NAOMI HAYASHIDA $^{2}$ and SUSUMU EGUCHI ${ }^{1}$ \\ ${ }^{1}$ Department of Surgery, Nagasaki University Graduate School of Biomedical Sciences, Nagasaki, Japan; \\ ${ }^{2}$ Division of Strategic Collaborative Research, Center for Promotion of Collaborative Research on Radiation and \\ Environment Health Effects, Atomic Bomb Disease Institute, Nagasaki University, Nagasaki, Japan
}

\begin{abstract}
Background/Aim: Docetaxel often induces peripheral neuropathy $(P N)$. The aim of this study was to elucidate the relationship between $P N$ and systemic inflammation parameters, namely the neutrophil-to-lymphocyte ratio (NLR), platelet-to-lymphocyte ratio (PLR), and monocyteto-lymphocyte ratio (MLR). Patients and Methods: We retrospectively evaluated 67 patients with breast cancer who were treated with docetaxel. Thirty patients (44.8\%) had received previous chemotherapy including anthracycline. Results: Overall, 51 patients (76.1\%) experienced PN. All the parameters increased with the number of administered courses of docetaxel. In an analysis of patients without previous chemotherapy, those suffering from $P N$ had a significantly higher NLR at the first and third cycles than those not suffering from $P N(2.9$ vs. 2.0, and 3.1 vs. 2.6, respectively, both $p<0.05)$, and the MLR at the first cycle was also significantly higher in those with PN than in those without (0.18vs. 0.15, $p<0.05)$. Conclusion: Systemic inflammation appears to contribute to the occurrence of $P N$ induced by docetaxel.
\end{abstract}

The taxane docetaxel promotes the stabilization of microtubules and causes $\mathrm{G}_{2} \mathrm{M}$ cell-cycle arrest and is one of the key drugs for treatment of breast cancer. Docetaxel for adjuvant purposes in patients with breast cancer improves the recurrence-free and overall survival $(1,2)$. In addition, docetaxel has been shown to help extend overall survival in patients with metastatic breast cancer (3). However, peripheral neuropathy (PN) is a major adverse effect associated with the administration of docetaxel,

Correspondence to: Kosho Yamanouchi, Department of Surgery, Nagasaki University Graduate School of Biomedical Sciences, 1-71 Sakamoto, Nagasaki, 852-8501, Japan. Tel: +81 958197316, Fax: +81958497319, e-mail: ymanouch@gk9.so-net.ne.jp

Key Words: Peripheral neuropathy, docetaxel, inflammation. with an any-grade frequency reported to range from 25.5-64.4\% $(1,3)$ and a grade $2-4$ frequency of $35 \%$ (4). PN induced by docetaxel sometimes leads to the need for dose reduction, a delayed schedule or the cessation of docetaxel altogether and reducing the quality of life of patients $(4,5)$. The exact mechanism underlying docetaxel-induced PN remains to be elucidated, and no definitive management for the prevention or treatment of this condition has been established.

Measures of systemic inflammatory responses, including the neutrophil-to-lymphocyte ratio (NLR), platelet-to-lymphocyte ratio (PLR), and monocyte-to-lymphocyte ratio (MLR), have been shown to be independent factors predictive of prognosis (6-8) or response to chemotherapy (9) in patients with various types of malignant tumors and of the occurrence or prognosis of non-malignant diseases $(10,11)$. Participation of the immune system or humoral factors, including cytokines or microenvironments, in such responses has been suggested. Recently, among patients with type 2 diabetes, those with higher NLR levels were found to be more likely to develop PN from diabetes than those with lower levels (12). Furthermore, in animal experiments, the activation of macrophages, which differentiate from monocytes, contributed to the induction of painful PN by paclitaxel, another taxane (13). We therefore hypothesized that PN induced by docetaxel is associated with systemic inflammation.

In the present study, we evaluated the relationship between the occurrence of $\mathrm{PN}$ in patients with breast cancer and systemic inflammation-based parameters, namely the NLR, PLR, and MLR, and determined whether or not they could function as predictive or causal factors for PN.

\section{Patients and Methods}

We reviewed the clinical and laboratory data of 67 female patients with primary invasive breast cancer diagnosed based on the findings of a biopsy or surgical specimens who had received docetaxel for at least four cycles as adjuvant/neoadjuvant therapy or first-line chemotherapy 
Table I. Morbidity rate, onset, and grade of docetaxel-induced peripheral neuropathy $(P N)$.

\begin{tabular}{lcc}
\hline Factor & $\mathrm{n}(\%)$ & $p$-Value \\
\hline Patients suffering from PN & $51(76.1 \%)$ & \\
Without previous chemotherapy & $27(73.0 \%)$ & 0.35 \\
With previous chemotherapy & $24(80.0 \%)$ & \\
Without anti-HER2 & $22(71.0 \%)$ & 0.26 \\
With anti-HER2 & $29(80.6 \%)$ & \\
Cycles with onset of PN & $23(45.1 \%)$ & NA \\
1 & $13(25.5 \%)$ & \\
2 & $7(13.7 \%)$ & \\
3 & $8(15.7 \%)$ & \\
4 & $33(64.7 \%)$ & \\
Highest grade of PN & NA \\
1 & $2(3.9 \%)$ & \\
2 & & \\
3 &
\end{tabular}

HER2: Human epidermal growth factor receptor 2, NA: not applicable.

for stage IV disease between January 2010 and December 2016 at our Department. Patients were excluded if they had already developed neuropathy from any cause, or taken any cytotoxic agents, or had hematological disorder. This retrospective study was approved by the Institutional Review Board of our institution (approval number 17101602), and the requirement to obtain informed consent was waived.

Protocol and evaluations. All of the patients received docetaxel at a dose of $75 \mathrm{mg} / \mathrm{m}^{2}$ intravenously every 3 weeks for four cycles. In all cases with docetaxel administered for adjuvant or neoadjuvant purpose, four cycles of epirubicin (E) with cyclophpsphamide (C) with or without 5-fluorouracil $(F)$ were given sequentially before or after docetaxel. Patients with human epidermal growth factor receptor 2 (HER2)-positive breast cancer received anti-HER2 agents, i.e. trastuzumab (initial dose $8 \mathrm{mg} / \mathrm{kg}$; loading dose $6 \mathrm{mg} / \mathrm{kg}$ ), and in stage IV cases, pertuzumab (initial dose $840 \mathrm{mg} / \mathrm{kg}$; loading dose $420 \mathrm{mg} / \mathrm{kg}$ ) was administered before docetaxel in each cycle. The adverse effects of docetaxel, including PN, fatigue, edema, and hand-foot syndrome, were evaluated using Common Terminology Criteria for Adverse Events (CTCAE) version 4.0 (14) and hematological examinations, including a complete blood count and differential leukocytes, in each cycle before treatment (baseline) and on day 21 of each cycle.

The NLR was defined as the absolute neutrophil count in the peripheral blood divided by the absolute lymphocyte count. The PLR was defined as the absolute platelet count in the peripheral blood divided by the absolute lymphocyte count. The MLR was defined as the absolute monocyte count in the peripheral blood divided by the absolute lymphocyte count.

Statistical analyses. The data are presented as the median (range) unless otherwise mentioned. The Mann-Whitney $U$-test was used for comparisons of continuous variables between two groups. Wilcoxon's signed-rank test was performed for comparisons between pretreatment and posttreatment inflammation-based parameters. Fisher's exact probability test was used for categorical variables. All of the statistical analyses were performed using the StatMate III software program for Macintosh (ATMS Co., Ltd., Tokyo, Japan).

\section{Results}

Initially, we reviewed the medical records of all patients with breast cancer who received docetaxel for adjuvant/ neoadjuvant therapy or first-line chemotherapy for stage IV between January 2010 and December 2016 at our Department. We excluded 13 patients for various reasons as follows: docetaxel was administered for fewer than four cycles due to adverse events in seven patients (allergic reaction in four, hand-foot syndrome in two, and interstitial pneumonia in one, but none due to $\mathrm{PN}$ ), differential leukocytes were not evaluated in four patients, chemotherapy had been administered for gynecological cancer previously in one patient, and a hematological disorder was detected in one patient. We, therefore, ultimately evaluated 67 patients.

The median age of the patients was 55 (36-74) years of age. A total of 63 patients $(94.0 \%)$ were administered docetaxel for adjuvant or neoadjuvant purposes, and four (6.0\%) for stage IV breast cancer. In the 63 patients who took it for adjuvant or neoadjuvant purposes, 30 were administered previous EC or FEC followed by docetaxel, and 33 docetaxel followed by EC or FEC (i.e. no previous chemotherapy). Overall, 30 patients (44.8\%) were administered previous chemotherapy. Thirty-six patients $(53.7 \%)$ received anti-HER2 agents concurrently with docetaxel. With regard to adverse events, edema of grade 1 or above was observed in $33(49.3 \%)$ patients, fatigue in 48 (71.6\%), and hand-foot syndrome in 25 (37.3\%). Three $(4.5 \%)$ patients required a dose reduction of docetaxel, and $6(9.0 \%)$ had their docetaxel cycles delayed for reasons other than PN.

As shown in Table I, PN of any grade was observed in 51 patients $(76.1 \%)$, and the proportion of patients that experienced it did not differ markedly based on previous use of chemotherapy or concurrent administration of anti-HER2 agents (both $p>0.05$ ). Most patients experienced at least grade $1 \mathrm{PN}$ from the first cycle.

Figure 1 shows the changes in the inflammation-based parameters with the cycles of docetaxel. All three parameters increased with the number of docetaxel cycles and had significantly higher values at the third cycle than before the administration of docetaxel [NLR: 2.0 (0.8-4.6) to 3.6 (1.1-6.9), $p<0.001$; PLR: 14.5 (1.4-51.6) to 19.0 (9.4-43.3), $p<0.001$; MLR: $0.18(0.06-0.61)$ to $0.30(0.11-1.23), p<0.001)$ (Figure 1). Figure 2 shows the relationship between the occurrence of $\mathrm{PN}$ and inflammation-based parameters. In the evaluation of all of the patients, there were no correlations between the NLR, PLR, or MLR before or at the first or third cycle of docetaxel and the occurrence of PN. However, in the analysis of only patients without previous chemotherapy, those with PN had a significantly higher NLR at the first and third cycles than those without PN [2.9 (1.6-6.9) vs. 2.0 (1.2-3.2), p=0.03; 3.1 (2.1-6.9) vs. 2.6 (1.3-3.7), $p=0.03$ ] and the MLR at the first cycle was also higher in those with PN than in those without [0.18 (0.13$0.46)$ vs. $0.15(0.12-0.27), p=0.04]$. There were no significant 

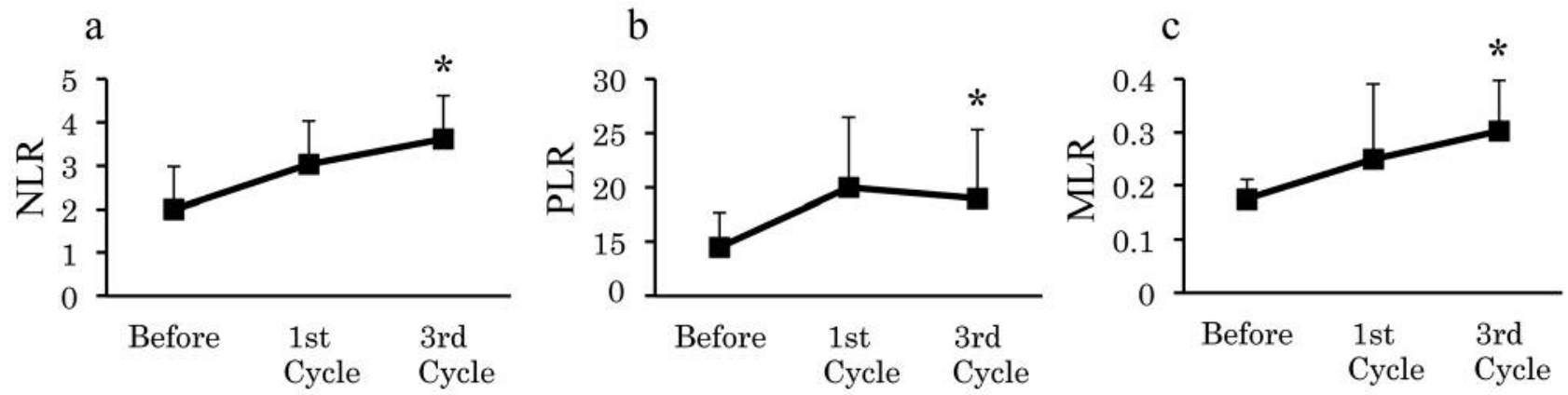

Figure 1. Inflammation-based parameters neutrophil-to-lymphocyte ratio $(N L R ; a)$, platelet-to-lymphocyte ratio (PLR; b), monocyte-to-lymphocyte ratio $(M L R ; c)$ according to docetaxel cycle. The value of each parameter increased with the number of docetaxel cycles. The data are presented as the median \pm quartile deviation. *Significantly different at $p<0.0001$ compared to the value before docetaxel administration.

a

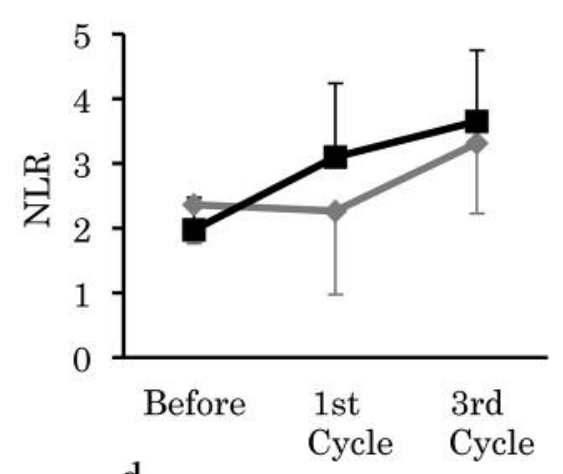

d

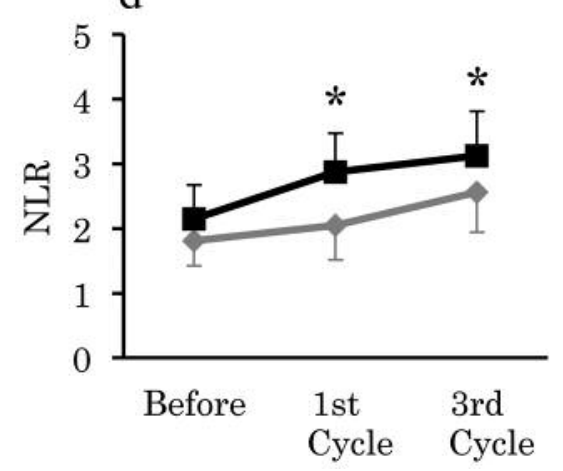

b

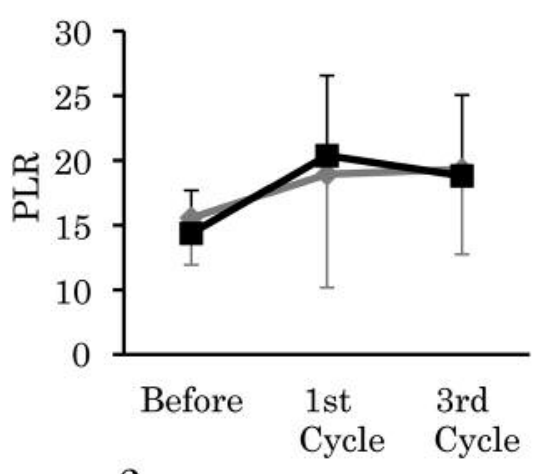

$\mathrm{e}$

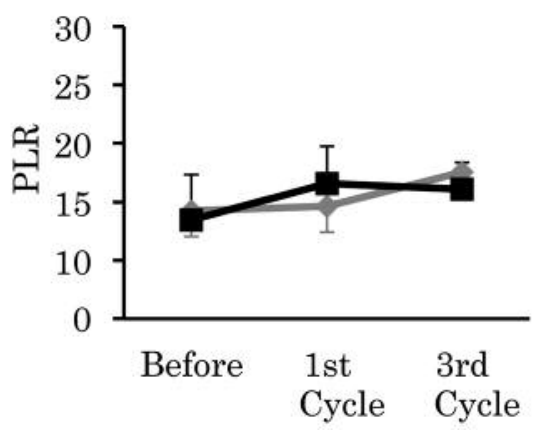

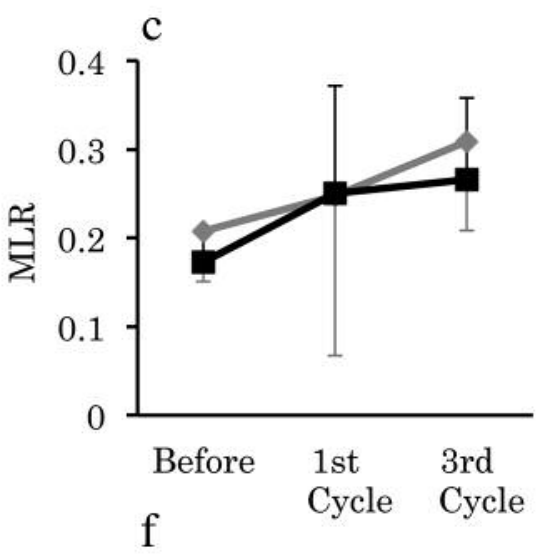

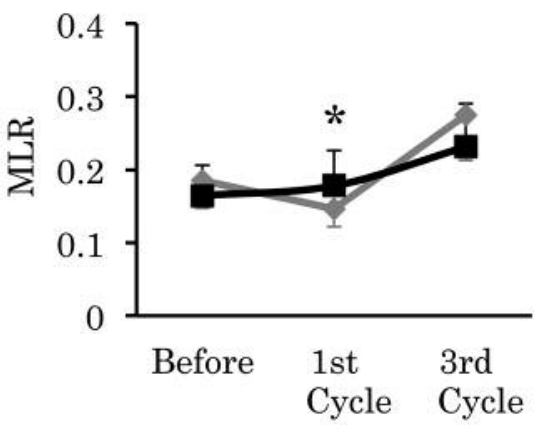

Figure 2. The relationship between the occurrence of docetaxel-induced peripheral neuropathy and inflammation-based parameters neutrophil-tolymphocyte ratio $(N L R ; a, d)$, platelet-to-lymphocyte ratio $(P L R ; b, e)$, and monocyte-to-lymphocyte ratio $(M L R ; c, f)$ in all patients $(a-c)$, and in patients without previous chemotherapy $(d-f)$. The data are presented as the median \pm quartile deviation. $*$ Significantly different at $p<0.05$ between the patients with and without docetaxel-induced peripheral neuropathy.

correlations between any inflammation-based parameter and the grade of docetaxel-induced PN (data not shown).

The NLR at the first cycle of docetaxel had no relationship with the occurrence of adverse events other than PN (i.e. edema, fatigue and hand-hoot syndrome; Table II), nor did the PLR or MLR (data not shown).

\section{Discussion}

In this retrospective study, we showed that inflammationbased parameters, including the NLR, PLR, and MLR, gradually increased with the number of cycles of docetaxel. Furthermore, systemic inflammation was deemed likely to 
Table II. Relationship between the occurrence of edema, fatigue, hand-foot syndrome and the neutrophil-to-lymphocyte ratio at the first cycle of docetaxel.

\begin{tabular}{|c|c|c|c|c|}
\hline \multirow[b]{2}{*}{ Adverse event } & \multirow[b]{2}{*}{ Patient group } & \multicolumn{2}{|c|}{ NLR according to severity of adverse advent } & \multirow[b]{2}{*}{$p$-Value } \\
\hline & & None & Grade $\geq 1$ & \\
\hline \multirow[t]{2}{*}{ Edema } & All patients & $2.8(1.2-7.0)$ & $3.2(1.2-13.5)$ & 0.20 \\
\hline & Without previous chemotherapy & $2.3(1.2-5.2)$ & $2.8(1.6-6.9)$ & 0.51 \\
\hline \multirow[t]{2}{*}{ Fatigue } & All patients & $3.2(1.2-13.5)$ & $2.9(1.2-8.0)$ & 0.40 \\
\hline & Without previous chemotherapy & $2.4(1.2-3.4)$ & $2.6(1.3-6.9)$ & 0.52 \\
\hline \multirow[t]{2}{*}{ Hand-foot syndrome } & All patients & $3.0(1.2-8.0)$ & $3.2(1.7-13.5)$ & 0.13 \\
\hline & Without previous chemotherapy & $2.3(1.2-3.7)$ & $2.6(1.7-6.9)$ & 0.52 \\
\hline
\end{tabular}

NLR: Neutrophil-to-lymphocyte ratio.

be involved in the occurrence of docetaxel-induced $\mathrm{PN}$ in patients without previous chemotherapy.

Systemic inflammation has been shown to be a predictive factor of prognosis in patients with malignancies (6-8) and cardiovascular disorders (15). In patients with type 2 diabetes, chronic inflammation was reported to be associated with diabetic complications (16), and notably, an increase in the NLR was associated with the occurrence and progression of diabetes-associated PN (12).

In animal experiments, PN induced by paclitaxel was characterized by neuroinflammation, as evidenced by the infiltration of macrophages, which are differentiated from monocytes, to sensory neurons and their supporting cells as well as secreted cytokines, resulting in neuronal apoptosis (13, 17, 18). The chemokine $\mathrm{C}-\mathrm{X} 3-\mathrm{C}$ motif chemokine ligand 1 contributed to this activation and infiltration of macrophages (13). In the present study, inflammation-based parameters increased with the number of cycles of docetaxel, suggesting that systemic inflammation may have some association with the occurrence of docetaxel-induced PN. The involvement of oxidative stress with docetaxel and other kinds of chemotherapy-induced PN $(5,19)$ as well as with diabetic PN (20) has already been reported. Since neutrophils are able to release reactive oxygen species and thereby induce oxidative stress (21), an absolute or relative increase in the numbers of neutrophils might contribute to PN.

Although several predictive factors of docetaxel-induced PN have been suggested, including pre-existing neuropathy (4), a body mass index $\geq 30 \mathrm{~kg} / \mathrm{m}^{2}$ (5), cumulative dose (3), and genetic variability (5), its underlying mechanisms remain largely unknown. The implementation of compression or cooling of limbs, or the administration of medications in order to prevent chemotherapy-induced PN has resulted in partial effectiveness $(22,23)$. Our findings will be of greater help in clarifying the mechanisms underlying chemotherapyinduced PN than in identifying predictive factors, as the baseline values of the inflammation-based parameters were identical, regardless of the occurrence of PN.
Why the occurrence of PN was associated with systemic inflammation-based parameters only in the evaluation of patients without previous chemotherapy is unclear at present. Although some changes in the inflammatory status due to previous chemotherapy might affect the morbidity from PN, the values of NLR before docetaxel administration were relatively unchanged, irrespective of previous chemotherapy (data not shown). As such chemotherapy including anthracycline has been reported to affect subsequent taxaneinduced PN (24), factors other than inflammatory parameters might influence docetaxel-induced PN.

Our study was associated with certain limitations. Firstly, this study was conducted on a small number of patients. Secondly, we only reviewed the phenomenon and speculated on the relation between systemic inflammation-based parameters and docetaxel-induced PN. In order validate our results, a prospective observational clinical study with a large number of participants is needed, along with measurements of cytokines or oxidants and antioxidants. Furthermore, animal experiments with reported models of chemotherapy-induced PN $(17,18)$ will be helpful for elucidating the exact mechanisms involved.

In our study, we investigated docetaxel because this is the most frequently used taxane in patients with breast cancer at our Department. Although more than half of the patients experienced PN induced by docetaxel, only $3.9 \%$ of cases showed grade 3 or worse severity, and no patients ceased docetaxel due to PN. However, paclitaxel and nanoparticle albumin-bound paclitaxel, which are also taxanes, are known to induce PN at a higher rate and with greater severity than docetaxel, and the underlying mechanisms of PN should be similar for these cases. Our results and further investigation with docetaxel are therefore expected to be applicable to paclitaxel and nab-paclitaxel, as well as other kinds of microtubule inhibitors, such as eribulin mesylate. Our findings may, therefore, help improve the efficacy of therapy and the quality of life in patients with breast cancer undergoing chemotherapy. 


\section{References}

1 Martin M, Pienkowski T, Mackey J, Pawlicki M, Guastalla JP, Weaver C, Tomiak E, Al-Tweigeri T, Chap L, Juhos E, Guevin R, Howell A, Fornander T, Hainsworth J, Coleman R, Vinholes J, Modiano M, Pinter T, Tang SC, Colwell B, Prady C, Provencher L, Walde D, Rodriguez-Lescure A, Hugh J, Loret C, Rupin M, Blitz S, Jacobs P, Murawsky M, Riva A and Vogel C: Adjuvant docetaxel for node-positive breast cancer. N Engl J Med 352: 2302-2313, 2005.

2 Peto R, Davies C, Godwin J, Gray R, Pan HC, Clarke M, Cutter D, Darby S, McGale P, Taylor C, Wang YC, Bergh J, Di Leo A, Albain K, Swain S, Piccart $M$ and Pritchard K: Comparisons between different polychemotherapy regimens for early breast cancer: Meta-analyses of long-term outcome among 100,000 women in 123 randomised trials. Lancet 379: 432-444, 2012.

3 Jones SE, Erban J, Overmoyer B, Budd GT, Hutchins L, Lower E, Laufman L, Sundaram S, Urba WJ, Pritchard KI, Mennel R, Richards D, Olsen S, Meyers ML and Ravdin PM: Randomized phase III study of docetaxel compared with paclitaxel in metastatic breast cancer. J Clin Oncol 23: 5542-5551, 2005.

4 Eckhoff L, Knoop AS, Jensen MB, Ejlertsen B and Ewertz M: Risk of docetaxel-induced peripheral neuropathy among 1,725 danish patients with early-stage breast cancer. Breast Cancer Res Treat 142: 109-118, 2013.

5 Eckhoff L, Feddersen S, Knoop AS, Ewertz M and Bergmann TK: Docetaxel-induced neuropathy: A pharmacogenetic casecontrol study of 150 women with early-stage breast cancer. Acta Oncol 54: 530-537, 2015.

6 Takeuchi H, Kawanaka H, Fukuyama S, Kubo N, Hiroshige S and Yano T: Comparison of the prognostic values of preoperative inflammation-based parameters in patients with breast cancer. PLoS One 12: e0177137, 2017.

7 Proctor MJ, Morrison DS, Talwar D, Balmer SM, Fletcher CD, O'Reilly DS, Foulis AK, Horgan PG and McMillan DC: A comparison of inflammation-based prognostic scores in patients with cancer. A glasgow inflammation outcome study. Eur J Cancer 47: 2633-2641, 2011.

8 Liu B, Huang Y, Sun Y, Zhang J, Yao Y, Shen Z, Xiang D and $\mathrm{He}$ A: Prognostic value of inflammation-based scores in patients with osteosarcoma. Sci Rep 6: 39862, 2016.

9 Chen Y, Chen K, Xiao X, Nie Y, Qu S, Gong C, Su F and Song E: Pretreatment neutrophil-to-lymphocyte ratio is correlated with response to neoadjuvant chemotherapy as an independent prognostic indicator in breast cancer patients: A retrospective study. BMC Cancer 16: 320, 2016.

10 Celikbilek A, Ismailogullari S and Zararsiz G: Neutrophil to lymphocyte ratio predicts poor prognosis in ischemic cerebrovascular disease. J Clin Lab Anal 28: 27-31, 2014.

11 Kekilli M, Tanoglu A, Sakin YS, Kurt M, Ocal S and Bagci S: Is the neutrophil to lymphocyte ratio associated with liver fibrosis in patients with chronic hepatitis B? World J Gastroenterol 21: 5575-5581, 2015.

12 Liu S, Zheng H, Zhu X, Mao F, Zhang S, Shi H, Li Y and Lu B: Neutrophil-to-lymphocyte ratio is associated with diabetic peripheral neuropathy in type 2 diabetes patients. Diabetes Res Clin Pract 130: 90-97, 2017.

13 Huang ZZ, Li D, Liu CC, Cui Y, Zhu HQ, Zhang WW, Li YY and Xin WJ: Cx3CL1-mediated macrophage activation contributed to paclitaxel-induced DRG neuronal apoptosis and painful peripheral neuropathy. Brain Behav Immun 40: 155-165, 2014.

14 National Cancer Institute. Common Terminology Criteria for Adverse Events (CTCAE). Available from: URL:http:// evs.nci.nih.gov/ftp1/CTCAE/About.html. Accessed May, 17, 2010.

15 Cicek G, Acikgoz SK, Bozbay M, Altay S, Ugur M, Uluganyan $\mathrm{M}$ and Uyarel H: Neutrophil-lymphocyte ratio and plateletlymphocyte ratio combination can predict prognosis in patients with ST-segment elevation myocardial infarction undergoing primary percutaneous coronary intervention. Angiology 66: 441447, 2015.

16 Bluher M, Unger R, Rassoul F, Richter V and Paschke R: Relation between glycaemic control, hyperinsulinaemia and plasma concentrations of soluble adhesion molecules in patients with impaired glucose tolerance or type II diabetes. Diabetologia 45: 210-216, 2002.

17 Peters CM, Jimenez-Andrade JM, Jonas BM, Sevcik MA, Koewler NJ, Ghilardi JR, Wong GY and Mantyh PW: Intravenous paclitaxel administration in the rat induces a peripheral sensory neuropathy characterized by macrophage infiltration and injury to sensory neurons and their supporting cells. Exp Neurol 203: 42-54, 2007.

18 Liu CC, Lu N, Cui Y, Yang T, Zhao ZQ, Xin WJ and Liu XG: Prevention of paclitaxel-induced allodynia by minocycline: Effect on loss of peripheral nerve fibers and infiltration of macrophages in rats. Mol Pain 6: 76, 2010.

19 Areti A, Yerra VG, Naidu V and Kumar A: Oxidative stress and nerve damage: Role in chemotherapy induced peripheral neuropathy. Redox Biol 2: 289-295, 2014.

20 Hosseini A and Abdollahi M: Diabetic neuropathy and oxidative stress: Therapeutic perspectives. Oxid Med Cell Longev 2013: 168039, 2013.

21 Lentsch AB, Kato A, Yoshidome H, McMasters KM and Edwards MJ: Inflammatory mechanisms and therapeutic strategies for warm hepatic ischemia/reperfusion injury. Hepatology 32: 169-173, 2000.

22 Ohno T, Mine T, Yoshioka H, Kosaka M, Matsuda S, De Kerckhove M, De Kerckhove C, Irie J, Inoue K, Haraguchi M, Kitajima M, Shinichiro I, Tokai H, Tanaka T and Izumida R: Management of peripheral neuropathy induced by nab-paclitaxel treatment for breast cancer. Anticancer Res 34: 4213-4216, 2014.

23 Tsuyuki S, Senda N, Kanng Y, Yamaguchi A, Yoshibayashi H, Kikawa Y, Katakami N, Kato H, Hashimoto T, Okuno T, Yamauchi A and Inamoto T: Evaluation of the effect of compression therapy using surgical gloves on nanoparticle albumin-bound paclitaxel-induced peripheral neuropathy: A phase ii multicenter study by the Kamigata Breast Cancer Study Group. Breast Cancer Res Treat 160: 61-67, 2016.

24 Shimozuma K, Ohashi Y, Takeuchi A, Aranishi T, Morita S, Kuroi K, Ohsumi S, Makino H, Katsumata N, Kuranami M, Suemasu K, Watanabe T and Hausheer FH: Taxane-induced peripheral neuropathy and health-related quality of life in postoperative breast cancer patients undergoing adjuvant chemotherapy: N-SAS BC 02, a randomized clinical trial. Support Care Cancer 20: 3355-3364, 2012.

Received August 27, 2017 Revised September 21, 2017 Accepted September 25, 2017 